\title{
Top Properties and Rare Decays from the Tevatron
}

\author{
Arnulf Quadt ${ }^{12}$ \\ 1 Physikalisches Institut, Universität Bonn, Nußallee 12, D-53115 Bonn, Germany \\ 2 University of Rochester, New York, c/o Fermilab - P.O. Box 500, 60510, IL, USA
}

Received: date / Revised version: date

\begin{abstract}
The top quark is the most recently discovered quark. Relatively little is known about its properties so far. Due to its very large mass of about $175 \mathrm{GeV} / \mathrm{c}^{2}$, the top quark behaves differently from all other quarks and provides a unique environment for tests of the Standard Model. Furthermore, it is believed to yield sensitivity to physics beyond the Standard Model. This report discusses the latest measurements and studies of top quark properties and rare decays from the Tevatron in Run II.
\end{abstract}

PACS. PACS-key discribing text of that key - PACS-key discribing text of that key

\section{Introduction}

The top quark discovery in 1995 by the experiments CDF and $D \varnothing[1]$ defines the start of the exciting era of top quark physics at the Tevatron. After very successful upgrades of the $p \bar{p}$ collider Tevatron for higher beam energy and luminosity and of both experiments for faster readout and trigger electronics, better tracking and muon detection, data taking in Run II started in the year 2001. Since then, the Tevatron provided more than $1 \mathrm{fb}^{-1}$ of $p \bar{p}$ collision data at $\sqrt{s}=1.96 \mathrm{TeV}$ to each experiment. At present, up to $370 \mathrm{pb}^{-1}$ have been analyzed in top quark studies.

Top quark physics at the Tevatron can be divided into the following categories: 1) top quark production, 2) fundamental properties of the top quark, 3) top quark interactions to gauge bosons, 4) anomalous top quark production, 5) anomalous top quark decays, and 6) new physics in events with $t \bar{t}$ topology.

The first category, the top quark production, is studied via the measurements of the strong $t \bar{t}$ production cross section and the search for the electroweak single-top production, in the Standard Model (SM) expected to be around $7 \mathrm{pb}$ and $\approx 3 \mathrm{pb}$, respectively. Measurements of the $t \bar{t}$ production cross section have been performed in many different top quark decay modes. The results are found to be consistent between the two experiments, all channels and with the Standard Model (SM) expectation within a combined precision of $\approx 14 \%$ [2]. The corresponding data sets, quantitatively understood in terms of selection efficiency and signal and background contribution form the basis of all studies of properties and rare decays of the top quark. Single-top production is expected to be observed with $1-2 \mathrm{fb}^{-1}$ of data [3].

The other categories are discussed in turn in this document in Sections 2 to 6 . All limits are quoted at the $95 \%$ CL unless noted otherwise.
In the SM, assuming unitarity of the three-generation CKM matrix, the matrix element $\left|V_{t b}\right|$ is found to be essentially unity. Therefore, the top quark is expected to decay to a $W$-boson and a $b$-quark nearly $100 \%$ of the time. The $W$-boson subsequently decays either to a pair of quarks or a lepton-neutrino pair. Depending on the lepton or hadronic decay of the two $W$-bosons, the resulting event topologies of $t \bar{t}$ decays are classified as all-jets channel $(46.2 \%)$, lepton+jets $(\ell+$ jets $)$ channel $(43.5 \%)$, and dilepton $(\ell \ell)$ channel (10.3\%). Each decay topology contains at least two $b$-jets. While $\ell$ in the above classification refers to $e, \mu$, or $\tau$, most of the results to date rely on the $e$ and $\mu$ channels. Therefore, in what follows, $\ell$ will be used to refer to $e$ or $\mu$, unless noted otherwise.

\section{Top Quark Interactions to Gauge Bosons}

\subsection{Spin Correlation}

$\mathrm{D} \emptyset$ has searched for evidence of spin correlation of $t \bar{t}$ pairs [5]. The $t$ and $\bar{t}$ are expected to be unpolarized but to be correlated in their spins. Since top quarks decay before hadronizing, their spins at production are transmitted to their decay daughter particles. Spin correlation is studied by analyzing the joint decay angular distribution of one $t$ daughter and one $\bar{t}$ daughter. The sensitivity to top spin is greatest when the daughters are down-type fermions (charged leptons or $d$-type quarks), in which case, the joint distribution is

$$
\frac{1}{\sigma} \frac{d^{2} \sigma}{d\left(\cos \theta_{+}\right) d\left(\cos \theta_{-}\right)}=\frac{1+\kappa \cdot \cos \theta_{+} \cdot \cos \theta_{-}}{4},
$$

where $\theta_{+}$and $\theta_{-}$are the angles of the daughters in the top rest frames with respect to a particular spin quantization axis, the optimal choice being the off-diagonal basis. 
Table 1. Measurements and lower limits of $R=B(t \rightarrow$ $W b) / B(t \rightarrow W q)$ and $\left|V_{t b}\right|$ from $\mathrm{CDF}$ and $\mathrm{D} \varnothing$.

\begin{tabular}{llc}
\hline$R$ or $\left|V_{t b}\right|$ & Source & $\int \mathcal{L} \mathrm{dt}\left(\mathrm{pb}^{-1}\right)$ \\
\hline$R=1.12_{-0.23}^{+0.27}$ & CDF Run II [6] & 160 \\
$R>0.61$ & CDF Run II [6] & 160 \\
$R=1.03_{-0.17}^{+0.19}$ & DØ Run II [7] & 230 \\
$R>0.64$ & DØ Run II [7] & 230 \\
$\left|V_{t b}\right|>0.78$ & CDF Run II [6] & 160 \\
$\left|V_{t b}\right|>0.80$ & DØ Run II [7] & 230 \\
\hline
\end{tabular}

In this basis, the SM predicts maximum correlation with $\kappa=0.88$ at the Tevatron. In Run I, D $\varnothing$ analyzed six dilepton events and obtained a likelihood as a function of $\kappa$, which weakly favored the SM $(\kappa=0.88)$ over no correlation $(\kappa=0)$ or anti-correlation $(\kappa=-1$, as would be expected for $t \bar{t}$ produced via an intermediate scalar). DØ quotes a limit $\kappa>-0.25$ at $68 \%$ CL. With improved statistics in the ongoing Run II analyses, an observation of $t \bar{t}$ spin correlation would support that the top quark decays before hadronization and allow further test of the QCD production mechanism.

\subsection{Measurement of $B(t \rightarrow W b) / B(t \rightarrow W q)$}

$\mathrm{CDF}$ and D $\varnothing$ report direct measurements of the $t \rightarrow W b$ branching ratio $[6,7]$. Comparing the number of events with 0,1 and 2 tagged $b$ jets in the lepton+jets channel, and for CDF also in the dilepton channel, and using the known $b$-tagging efficiency, the ratio $R=B(t \rightarrow$ $W b) / \sum_{q=d, s, b} B(t \rightarrow W q)$ can be extracted (Figure 1). $\mathrm{D} \varnothing$ performs a simultaneous fit for the production cross section $\sigma_{t \bar{t}}$ and the ratio $R$. A deviation of $R$ from unity would imply either non-SM top decay, a non-SM background to $t \bar{t}$ production, or a fourth generation of quarks. Assuming that all top decays have a $W$ boson in the final state, that only three generations of fermions exist, and that the CKM matrix is unitary, CDF and DØ also extract the CKM matrix-element $\left|V_{t b}\right|$. The results of these measurements are summarized in Table 1. The top quark decay to $W b$ is indeed found to be dominant, although these studies are presently limited by statistics and will profit from the upcoming larger data sets.

A more direct measurement of the $W t b$ coupling constant will be possible when enough data are accumulated to detect the $s$-channel and $t$-channel single-top production processes [3]. The cross sections for these processes are proportional to $\left|V_{t b}\right|^{2}$, and no assumption is needed on the number of families or on the unitarity of the CKM matrix in extracting $\left|V_{t b}\right|$.

\subsection{Study of $B(t \rightarrow \tau \nu q)$}

The SM's heavy third generation particles, the top and bottom quarks, the tau and the tau neutrino are intriguing. The high energies required to produce the third generation particles, particularly in the case of the top quark,

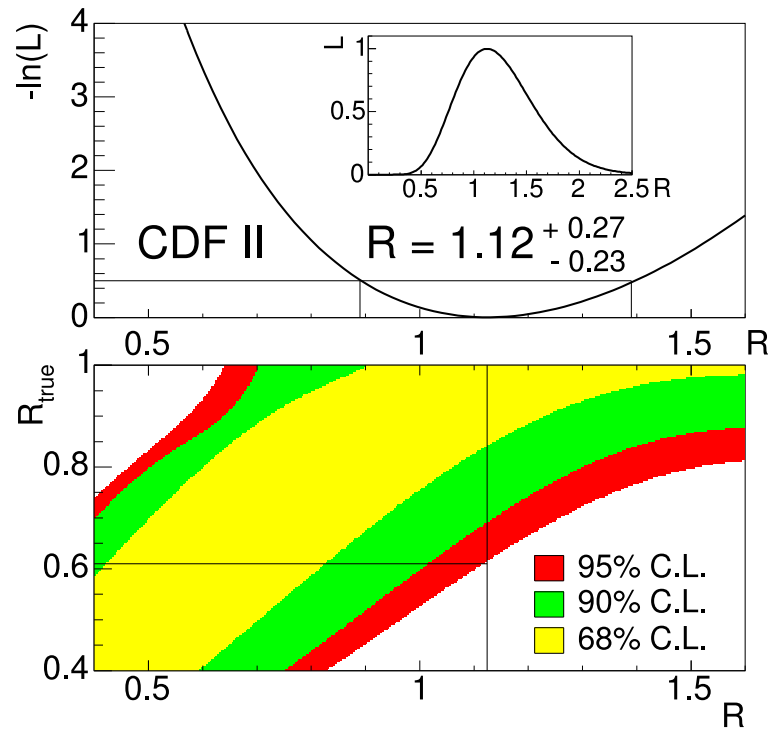

Fig. 1. Top: CDF likelihood as a function of $R$ (inset) and its negative logarithm. Bottom: Confidence level bands for $R_{\text {true }}$ as a function of $R$. The measurements of $R=1.12$ (vertical line) implies $R>0.61$ (horizontal line).

have resulted in the particles being the least studied in the SM. Current measurements leave room for new physics in the interactions and decays of these particles. The high masses of the particles give rise to the hope that studying them could help shed light on the origin of fermion masses. CDF measures the rate of top-antitop events with a semileptonically decaying tau in $t \bar{t} \rightarrow e \tau b b \nu \nu$ and $t \bar{t} \rightarrow \mu \tau b b \nu \nu$ events in $200 \mathrm{pb}^{-1}$ of Run II data [8]. Semi-leptonic tau decays account for $64 \%$ of all tau decays. This analysis does not include taus decaying to electrons or muons because their leptonic tau decays are difficult to differentiate from prompt leptons. CDF compares the observed with the predicted rate as a test of the SM. Many extensions to the SM predict identical final states which could lead to an anomalous rate. For example the charged Higgs decay from $t \bar{t}, t \bar{t} \rightarrow H^{ \pm} W b \bar{b}, H^{ \pm} \rightarrow \tau^{ \pm} \nu_{\tau}$. This analysis is a search for any such anomalous processes that could show up in the final state as an enhanced (or suppressed) rate for tau leptons in top decays. The ratio $r_{\tau} \equiv B(t \rightarrow b \tau \nu) / B_{S M}(t \rightarrow b \tau \nu)$ is found to be $r_{\tau}<5.0$ and therefore consistent with the SM.

\subsection{Measurement of the Helicity of the $W$-Boson in Top Quark Decays}

Studies of decay angular distributions provide a direct check of the $V-A$ nature of the $W t b$ coupling and information on the relative coupling of longitudinal and transverse $W$ bosons to the top quark. In the SM, the fraction of decays to longitudinally polarized $W$ bosons is expected to be $\mathcal{F}_{0}^{\mathrm{SM}}=x /(1+x), x=m_{t}^{2} / 2 M_{W}^{2}\left(\mathcal{F}_{0}^{\mathrm{SM}} \sim 70 \%\right.$ for $\left.m_{t}=175 \mathrm{GeV} / c^{2}\right)$. Fractions of left- or right-handed $W$ bosons are denoted as $\mathcal{F}_{-}$and $\mathcal{F}_{+}$, respectively. In the $\mathrm{SM}, \mathcal{F}_{-}$is expected to be $\approx 30 \%$ and $\mathcal{F}_{+} \approx 0 \%$. CDF 
Table 2. Measurement and upper limits of the $W$ helicity in top quark decays from $\mathrm{CDF}$ and $\mathrm{D} \varnothing$. The integrated luminosity $\int \mathcal{L} \mathrm{dt}$ is given in units of $\left(\mathrm{pb}^{-1}\right)$.

\begin{tabular}{lllc}
\hline$W$ helicity & Source & $\int \mathcal{L} \mathrm{dt}$ & Method \\
\hline $\mathcal{F}_{0}=0.91 \pm 0.39$ & CDF Run I [9] & 106 & $p_{T}^{\ell}$ \\
$\mathcal{F}_{0}=0.56 \pm 0.32$ & DØ Run I [10] & 125 & ME \\
$\mathcal{F}_{0}=0.74_{-0.34}^{+0.22}$ & CDF Run II [11] & 200 & $M_{\ell b}^{2}+p_{T}^{\ell}$ \\
\hline $\mathcal{F}_{+}<0.18$ & CDF Run I [12] & 110 & $M_{\ell b}^{2}+p_{T}^{\ell}$ \\
$\mathcal{F}_{+}<0.27$ & CDF Run II [11] & 200 & $M_{\ell b}^{2}+p_{T}^{\ell}$ \\
$\mathcal{F}_{+}<0.25$ & DØ Run II [13] & $230-370$ & $\cos \theta^{*}+p_{T}^{\ell}$ \\
\hline
\end{tabular}

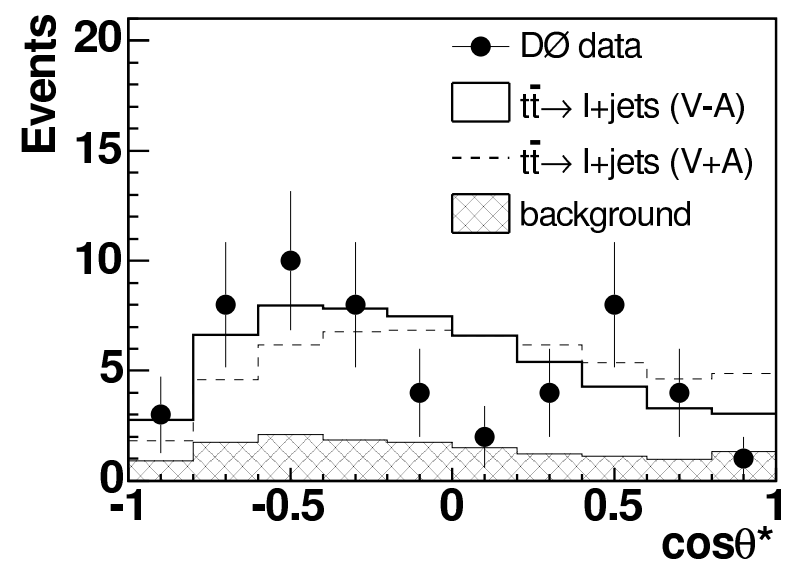

Fig. 2. $\cos \theta^{*}$ distribution observed in the $\mathrm{D} \varnothing$ data along with the SM prediction (solid line) and a model with a pure $V+A$ interaction (dashed line) for the $b$-tagged lepton + jets sample.

and $\mathrm{D} \varnothing$ use various techniques to measure the helicity of the $W$ boson in top quark decays in lepton + jets events. The first method uses a kinematic fit, similar to that used in the lepton+jets mass analyses [4], but with the top quark mass constrained to $175 \mathrm{GeV} / \mathrm{c}^{2}$, to improve the reconstruction of final state observables and choose the assignment to quarks and leptons as that with the lowest $\chi^{2}$. The distribution of the helicity angle $\left(\cos \theta^{*}\right)$ between the lepton and the $b$ quark in the $W$ rest frame, provides the most direct measure of the $W$ helicity (Figure 2). The second method $\left(p_{T}^{\ell}\right)$ uses the different lepton $p_{T}$ spectra from longitudinally or transversely polarized $W$-decays to determine the relative contributions. This method is also used by both experiments in the dilepton channel. A third method uses the invariant mass of the lepton and the $b$-quark in top decays $\left(M_{\ell b}^{2}\right)$ as an observable, which is directly related to $\cos \theta^{*}$. Finally, the Matrix Element method (ME), initially developed for the top quark mass measurement, has also been used, forming a 2-dimensional likelihood $\mathcal{L}\left(m_{\text {top }}, \mathcal{F}_{0}\right)$, where the mass-dependence is integrated out so that only the sensitivity to the $W$-helicity in the top quark decay is exploited. The results of all CDF and $\mathrm{D} \emptyset$ analyses, summarized in Table 2 , are in agreement with the SM expectation, but within large statistical uncertainties.

\subsection{Search for Top Quark Decay via FCNC Couplings}

Physics beyond the SM can manifest itself by altering the expected rate of flavor-changing neutral-current (FCNC) interactions. FCNC decays of the top quark are of particular interest. The large mass of the top quark suggests a strong connection with the electroweak symmetry breaking sector. Evidence for unusual decays of the top quark might provide insights into that mechanism. For the top quark, the FCNC decays $t \rightarrow q Z$ and $t \rightarrow q \gamma$ (where $q$ denotes either a $c$ - or a $u$-quark) are expected to be exceedingly rare (branching fractions of $10^{-10}$ or smaller), since they are suppressed by the GIM mechanism and any observation of these decays in the available data sample would indicate new physics. In general, FCNC interactions are present in models which contain an extended Higgs sector, Supersymmety, dynamical breaking of the electroweak symmetry, or an additional symmetry.

CDF reported a search for flavor changing neutral current (FCNC) decays of the top quark $t \rightarrow q \gamma$ and $t \rightarrow q Z$ in the Run I data [14]. CDF assumes that one top decays via FCNC while the other decays via $W b$. For the $t \rightarrow q \gamma$ search, two signatures are examined, depending on whether the $W$ decays leptonically or hadronically. For leptonic $W$ decay, the signature is $\gamma \ell$ and missing $E_{T}$ and two or more jets, while for hadronic $W$ decay, it is $\gamma+\geq 4$ jets. In either case, one of the jets must have a secondary vertex b tag. One event is observed $(\mu \gamma)$ with an expected background of less than half an event, giving an upper limit on the top branching ratio of $B(t \rightarrow q \gamma)<3.2 \%$. In the search for $t \rightarrow q Z$, CDF considers $Z \rightarrow \mu \mu$ or ee and $W \rightarrow q q^{\prime}$, giving a $Z+$ four jets signature. One $\mu \mu$ event is observed with an expected background of 1.2 events, giving an upper limit on the top branching ratio of $B(t \rightarrow q Z)<0.33$. These limits on top quark decay branching ratios can be translated into limits on the flavor-changing neutral current couplings $\kappa_{\gamma}<0.42$ and $\kappa_{Z}<0.73$. With $2 \mathrm{fb}^{-1}$, CDF and DØ are expected to improve their sensitivity to $\kappa_{\gamma}$ and to $\kappa_{Z}$ significantly with the increased Run II data set.

\section{Fundamental Properties of the Top Quark}

\subsection{Top Quark Mass}

The Tevatron Electroweak Working Group has recently combined all available direct measurements of the top quark mass yielding a new world average of $m_{\text {top }}=172.7 \pm$ $2.9 \mathrm{GeV} / \mathrm{c}^{2}[4,15]$. The ultimate precision from the Tevatron on the top mass measurement is expected to be better than $2.0 \mathrm{GeV} / \mathrm{c}^{2}$ per experiment.

\subsection{Electric Charge of the Top Quark}

The top quark is the only quark whose electric charge has not been measured through a production threshold in $e^{+} e^{-}$collisions. Since the CDF and $\mathrm{D} \varnothing$ analyses on top quark production do not associate the $b, \bar{b}$ and $W^{ \pm}$ 
uniquely to the top or antitop, decays such as $t \rightarrow W^{+} \bar{b}$, $\bar{t} \rightarrow W^{-} b$ are certainly conceivable. A charge $4 / 3$ quark of this kind would be consistent with current electroweak precision data. The $Z \rightarrow \ell^{+} \ell^{-}$and $Z \rightarrow b \bar{b}$ data can be fitted with a top quark of mass $m_{t}=270 \mathrm{GeV} / \mathrm{c}^{2}$, provided that the right-handed $b$ quark mixes with the isospin $+1 / 2$ component of an exotic doublet of charge $-1 / 3$ and $-4 / 3$ quarks, $\left(Q_{1}, Q_{4}\right)_{R}$. CDF and D $\varnothing$ study the top quark charge in double-tagged lepton+jets events. Assuming the top and antitop quarks have equal but opposite electric charge, then reconstructing the charge of the $b$-quark through jet charge discrimination techniques, the $\left|Q_{t o p}\right|=4 / 3$ and $\left|Q_{t o p}\right|=2 / 3$ scenarios can be differentiated. CDF and D $\varnothing$ both have already collected sufficient data to obtain sensitivity to the $\left|Q_{t o p}\right|=4 / 3$ case. The analyses are ongoing, results are expected to be made public soon.

\section{Anomalous Top Quark Production}

\subsection{Cross Section Ratio $\sigma_{\ell \ell} / \sigma_{\ell+j e t s}$}

It is a priori not obvious, that the 'top quark', observed in the dilepton decay mode is identical to the 'top quark' in the lepton+jets decay mode. If both decay modes result exclusively from the decay of the SM top quark, they should have the same production cross section. If the production or the decay of the top quarks had non-SM contributions, one mode might be enhanced with respect to the other.

$\mathrm{CDF}$ has measured the cross section ratio $R_{\sigma}=\sigma_{l l} / \sigma_{l+j e t s}$ of the $t \bar{t}$ production cross section in the dilepton and the lepton+jets channels in $125 \mathrm{pb}^{-1}$ of Run II data. CDF finds $R_{\sigma}=1.45_{-0.55}^{+0.83}$ and $R_{\sigma}>$ $0.46(<4.45)$, consistent with the SM. This result is also translated into generic top decay branching ratio limits. The considered cases are a fully hadronic decay $t \rightarrow X b$, where $\operatorname{Br}(X \rightarrow q q)=100 \%$ or a fully leptonic decay, i.e. $t \rightarrow Y b$, where $\operatorname{Br}(Y \rightarrow q q)=100 \%$. The limits on $R_{\sigma}$ translate into limits on the fully hadronic or the fully leptonic decay of the top quark as $B r(t \rightarrow X b)<0.46$ and $B r(t \rightarrow Y b)<0.47$.

\subsection{Anomalous Kinematics in $t \bar{t}$ Events}

CDF reports a search for anomalous kinematics of $t \bar{t}$ dilepton events in $193 \mathrm{pb}^{-1}$ [17]. A new a priori technique has been developed, designed to isolate the subset of events in a data sample which reveals the largest deviation from SM expectation and to quantify the significance of this departure. Four variables are considered: the missing transverse energy, $E_{T}$, the transverse momentum of the leading lepton $p_{T}^{\ell}$, the angle $\phi_{\ell m}$ between the leading lepton and the direction of $E_{T}$ in the plane transverse to the beam, and a variable $T$, representing how well the kinematics of an event satisfy the $t \bar{t}$ decay hypothesis based on the expected and observed $E_{T}$ vector. This method is especially sensitive to data subsets that preferentially populate regions where new high- $p_{T}$ physics can be expected. No such subset is found. Although the lepton $p_{T}$ distribution exhibits a mild excess at low $p_{T}$, CDF determines the level of consistency of the $t \bar{t}$ dilepton sample with the SM expectation and finds a $p$-value of $1.0-4.5 \%$, showing good agreement with the SM.

This type of search for anomalous kinematics is presently statistics limited and will improve with larger data sets.

\subsection{Top Production via Intermediate Resonances}

Motivated by the large mass of the top quark, several models suggest that the top quark plays a role in the dynamics of electroweak symmetry breaking. One example is topcolor, where a large top quark mass can be generated through the formation of a dynamic $t \bar{t}$ condensate, $X$, which is formed by a new strong gauge force coupling preferentially to the third generation. Another example is topcolor-assisted technicolor, predicting a heavy $Z^{\prime}$ boson that couples preferentially to the third generation of quarks with cross sections expected to be visible at the Tevatron. CDF and D $\varnothing$ have searched for $t \bar{t}$ production via intermediate, narrow-width, heavy vector bosons $X$ in the lepton+jets channels. The $t$ and $\bar{t}$ final states are identified through a kinematic fit. The possible $t \bar{t}$ production via an intermediate resonance $X$ is sought for as a peak in the spectrum of the invariant $t \bar{t}$ mass. CDF and D $\varnothing$ exclude narrow width heavy vector bosons $X[18]$ with mass $M_{X}<480 \mathrm{GeV} / \mathrm{c}^{2}$ and $M_{X}<560 \mathrm{GeV} / \mathrm{c}^{2}$, respectively, in Run I [19], and $M_{X}<680 \mathrm{GeV} / \mathrm{c}^{2}$ in DØ Run II [20].

\section{Anomalous Top Quark Decays}

\subsection{Search for Charged Higgs Boson in $t \bar{t}$ Decays}

Both CDF and D $\varnothing$ have searched for non-SM top decays, particularly those expected in supersymmetric models, such as $t \rightarrow H^{+} b$, followed by $H^{+} \rightarrow \tau^{+} \bar{\nu}$ or $c \bar{s}$. The $t \rightarrow H^{+} b$ branching ratio has a minimum at $\tan \beta=$ $\sqrt{m_{t} / m_{b}} \simeq 6$, and is large in the region of either $\tan \beta \ll 6$ or $\tan \beta \gg 6$. In the former range, $H^{+} \rightarrow c \bar{s}$ is dominant, while $H^{+} \rightarrow \tau^{+} \bar{\nu}$ dominates in the latter range. These studies are based either on direct searches for these final states, or on top "disappearance". In the standard lepton+jets or dilepton cross section analyses, any charged Higgs decays are not detected as efficiently as $t \rightarrow W^{ \pm} b$, primarily because the selection criteria are optimized for the standard decays, and because of the absence of energetic isolated leptons in Higgs decays. A significant $t \rightarrow$ $H^{+} b$ contribution would give rise to measured $t \bar{t}$ cross sections lower than the SM prediction (assuming that non$\mathrm{SM}$ contributions to $t \bar{t}$ production are negligible).

In Run II, CDF has searched for charged Higgs production in dilepton, lepton+jets and lepton+hadronic tau final states, considering possible $H^{+}$decays to $c \bar{s}, \tau \bar{\nu}$, $t^{*} b$ or $W^{+} h^{0}$ in addition to the SM decay $t \rightarrow W^{+} b$ [21]. Depending on the top and Higgs decay branching 


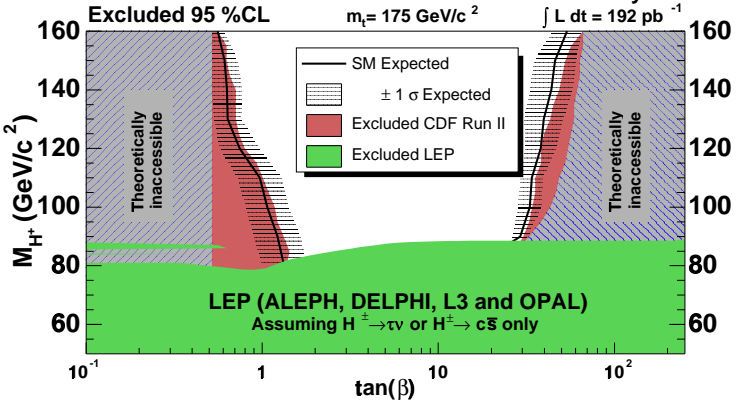

